\title{
Improved Signal Copy with Partially Known or Unknown Array Response
}

\author{
J. YANG, S. DaAs, AND A. SWindlehurst \\ Dept. of Elec. \& Comp. Engineering \\ Brigham Young University \\ Provo, UT 84602
}

\begin{abstract}
Blind adaptive algorithms extract signals that overlap in time and frequency by exploiting their temporal structure, but ignore any available spatial (array response) data. On the other hand, direction-finding based methods compute the signal copy weights using estimates of the signal directions, but ignore information about signal structure. In this paper, we present two simple iterative techniques that attempt to incorporate both temporal and spatial information in estimating the signal waveforms received by an array of sensors. The first technique asoumes an initial blind signal estimate is available, and uses least-squares to approximate the array response and refine the signal estimate. The second method is applicable to digitally modulated signals, and use bit decisions made on an initial signal estimate to recom. pute the signal copy weight vectors. A theoretical performance analysis of both algorithms is conducted for the high SNR case, and some representative simulation results are included.
\end{abstract}

\section{Introduction}

CONVENTIONAL techniques for signal copy that exploit the spatial coherence of the incoming signals can not be applied without accurate knowledge of the directions of arrival (DOAs) of the signals and the array response. In addition, such techniques make no assumption about the signals themselves, and consequently can not exploit any such available information. In response to these drawbacks, a number of blind adaptive signal copy algorithms have been proposed, such as SCORE [1] and the constant modulus approach [2]. These methods exploit known properties about the sig. nals (cyclostationarity, constant modulus, etc.) to al leviate the need for precise array calibration data. On the other hand, these algorithms may converge slowly (or in some cases not at all) and they have difficulty with certain types of signal scenarios.

Thus, conventional techniques based on direction finding (DF) do not employ any knowledge about the signal structure, and blind copy algorithms do not use

${ }^{1}$ This work was supported in part by a contract from $E$ Systems, Inc., Greenville Division (Dr. William A. Gardner, Principal Investigator), and by the Natioual Science Foundation under grant MIP-9110112 any knowledge of the array response. In many situations, information about both the array calibration and the signal structure is available (or can be estimated from the data), and it is obviously suboptimal to ignore either. In this paper, we present two simple suboptimal algorithms that attempt to improve signal copy performance by exploiting both the array and signal structure in an alternating fashion. The first method assumes a blind copy algorithm has been applied to obtain an initial estimate of the signal waveforms, and then uses LS methods to estimate the steering matrix and revise the signal estimates. The second technique assumes digitally modulated signals and is decision directed, in that bit decisions made on a preliminary signal estimate are used to generate a new set of copy weights, and an updated signal estimate.

In addition to presenting the algorithms and their implementation, we present a theoretical analysis of their performance for the high SNR case. The Iterative Blind/LS algorithm is shown to always reduce the mean-squared error of the initial signal estimate. In addition, an expression for the bit error rate of the decision directed algorithm is derived for the special case of BPSK signals. After some background material in the following section, the two algorithms are presented and analyzed in Sections 3 and 4, respectively. We note here some recent work described in [3] that closely parallels our own. The main difference is that, while [3] focused on parameter identifiability, we emphasize algorithm performance analysis.

\section{Data Model and Relevant Algorithms}

Consider an array of $m$ sensors, having arbitrary positions and characteristics, that receives the waveforms of $d$ narrowband (co-channel) signals from sources in the far-field of the array. The vector of complex sensor outputs is denoted $\mathbf{x}(t)$, and is modeled by the following familiar equation:

$$
\mathbf{x}(t)=\mathbf{A}(\boldsymbol{\theta}) \mathbf{s}(t)+\mathbf{n}(t) .
$$

The columns of the $m \times d$ matrix $\mathbf{A}$ are the so-called array steering vectors, and are denoted as $\mathbf{a}\left(\theta_{i}\right), i=$ $1, \ldots, d$. These vectors describe the array response to a 
unit waveform with DOA $\theta_{i}$. The $d$-vector $\mathrm{s}(t)$ is composed of the complex waveforms (in-phase and quadrature components) of the signals received at time $t$, and the $m$-vector $\mathbf{n}(t)$ accounts for additive measurement noise. The noise term is modeled as a zero-mean, stationary, complex random process that is uncorrelated with any of the signals. It is further assumed (without loss of generality) to be spatially and temporally white with variance $\sigma_{n}^{2}$

The general problem addressed in this paper is the estimation of the signal waveforms at $N$ distinct sample points $\mathbf{S}=[\mathbf{s}(1), \cdots, \mathbf{s}(N)]$, using the received data $\mathbf{X}$ :

$$
\mathbf{X}=\mathbf{A}(\boldsymbol{\theta}) \mathbf{S}+\mathbf{N}
$$

where $\mathbf{X}$ and $\mathbf{N}$ are defined similarly to $\mathbf{S}$. This is typically done by forming a linear combination of the array outputs, as in $\hat{\mathbf{S}}=\mathbf{W}^{*} \mathbf{X}$. The $i^{\text {th }}$ column of the matrix $\mathbf{W}$ is referred to as the signal copy weight vector (or sometimes beamformer weights) for the $i^{\text {th }}$ signal. There are a number of methods available for choosing the weight matrix $\mathbf{W}$, each with a different optimality criterion and a different set of assumptions about what a priori information is available. We mention two here, the least-squares (LS) and minimum mean-squared error (MMSE) approaches.

The LS algorithm finds the signal estimate that, in the LS sense, best matches the received data given an estimate of the steering matrix $\hat{\mathbf{A}}=\mathbf{A}(\hat{\boldsymbol{\theta}})$ :

$$
\mathbf{S}_{L S}=\arg \min _{S}\|\mathbf{X}-\hat{\mathbf{A}} \mathbf{S}\|_{F}^{2}=\hat{\mathbf{A}}^{\dagger} \mathbf{X},
$$

where in this case $\mathbf{W}_{L S}^{*}=\dot{\mathbf{A}}^{\dagger}=\left(\hat{\mathbf{A}}^{*} \hat{\mathbf{A}}\right)^{-1} \hat{\mathbf{A}}^{*}$. If the noise is temporally white and Gaussian, then it is easy to show that $\hat{\mathbf{S}}_{t s}$ corresponds to the maximum likelihood estimate of $\mathbf{S}$. The MMSE weight vector is calculated to be

$$
\mathbf{W}_{M S E}=\arg \min _{W} \mathcal{E}\left\{\left\|\mathbf{W}^{*} \mathbf{X}-\mathbf{S}\right\|_{F}^{2}\right\}=\mathbf{R}_{x x}^{-1} \mathbf{R}_{x} s,
$$

where $\mathbf{R}_{x x}$ is the covariance of the array data, and $\mathbf{R}_{w s}$ is the cross-covariance of $\mathbf{x}(t)$ and $\mathbf{s}(t)$. In this case, the weight vector depends on the signals themselves through $\mathbf{R}_{x s}$, and thus the MMSE method cannot be implemented directly. Typically, $\mathbf{R}_{x x}$ is replaced by a sample average, and $\mathbf{R}_{x s}$ is replaced hy some other suitable estimate.

\section{An Iterative Blind/LS Algorithm}

In the LS approach described above, $\hat{\mathbf{A}}=\mathbf{A}(\hat{\boldsymbol{\theta}})$ is typically obtained by first estimating the DOAs, though this is not necessary since there is no explicit dependence on the DOAs in (2). In particular, if $\mathbf{S}$ were known (or an estimate $\hat{\mathbf{S}}$ were available), one could minimize (2) with respect to $\hat{\mathbf{A}}$ to obtain

$$
\ddot{\mathbf{A}}=\mathbf{X} \mathbf{S}^{*}\left(\mathbf{S S}^{*}\right)^{-1}=\mathbf{X S}^{\dagger} \text {. }
$$

This set of estimated steering vectors could then be used to form the LS signal copy weights used in (2) The outline of an algorithm based on this idea is given below:

1. Use a blind copy algorithm to find a set of signal copy weights $\mathbf{W}$, and obtain an initial signal estimate $\hat{\mathbf{S}}=\mathbf{W}^{*} \mathbf{X}$.

2. Treating $\hat{\mathbf{S}}$ as a constant, minimize the $\mathbf{L S}$ cost function $\|\mathbf{X}-\mathbf{A} \hat{\mathbf{S}}\|_{F}^{2}$ with respect to $\mathbf{A}$ to obtain the following estimate of the steering matrix:

$$
\hat{\mathbf{A}}=\mathbf{X} \hat{\mathbf{S}}^{\dagger} \text {. }
$$

3. Treating $\hat{\mathbf{A}}$ as a constant, obtain a refined estimate of the signals using LS again:

$$
\hat{\hat{\mathbf{S}}}=\hat{\mathbf{A}}^{\dagger} \mathbf{X} \text {. }
$$

\subsection{Performance Analysis}

If we assume that $\tilde{\mathbf{S}}$ represents the error associated with the initial blind signal estimate $(\hat{\mathbf{S}}=\mathbf{S}+\tilde{\mathbf{S}})$, then the resulting error in the estimate of $\mathbf{A}$, denoted $\Delta \mathbf{A}$, can be approximated as

$$
\hat{\mathbf{A}}=\mathbf{A}+\Delta \mathbf{A} \simeq \mathbf{A}-\mathbf{A} \tilde{\mathbf{S}} \mathbf{S}^{\dagger}+\mathbf{N} \tilde{\mathbf{S}}^{*}\left(\mathbf{S S}^{*}\right)^{-1}
$$

to first order in $\tilde{\mathbf{S}}$. Note that we have also ignored terms involving the product $\mathrm{NS}^{*}$ since the signals and noise are assumed to be uncorrelated. Using similar firstorder arguments, the refined signal copy estimate may be approximately expressed as $\mathbf{S}+\tilde{\tilde{\mathbf{S}}}$, where

$\tilde{\tilde{\mathbf{S}}} \simeq \mathbf{A}^{\dagger} \mathbf{N}+\left(\mathbf{A}^{*} \mathbf{A}\right)^{-1} \Delta \mathbf{A}^{*} \mathbf{P}_{A}^{\perp} \mathbf{N}-\mathbf{A}^{\dagger} \Delta \mathbf{A} \mathbf{S}-\mathbf{A}^{\dagger} \Delta \mathbf{A} \mathbf{A}^{\dagger} \mathbf{N}$

and $\mathbf{P}_{A}^{\perp}=\mathbf{I}-\mathbf{A}\left(\mathbf{A}^{*} \mathbf{A}\right)^{-1} \mathbf{A}^{*}$.

The final signal copy error thus depends on the initial error in a rather complicated way. If we take the special case where the SNR is relatively high and the terms involving $\mathbf{N}$ above may be ignored, substituting (6) into (7) leads to

$$
\tilde{\tilde{\mathbf{S}}} \simeq-\mathbf{A}^{\dagger} \Delta \mathbf{A S} \simeq \tilde{\mathbf{S}} \mathbf{P}_{S^{*}},
$$

where $\mathbf{P}_{S}$ * is the projection onto the row space of $\mathbf{S}$. In such cases, we are thus guaranteed that $\|\tilde{\tilde{\mathbf{S}}}\| \leq\|\tilde{\mathbf{S}}\|$, and hence that the LS iteration in steps 2 and 3 will improve the mean squared error (MSE) of the signal estimate. This also implies that repeating steps 2 and 3 will not lead to further improvement, since to first order,

$$
\tilde{\tilde{\mathbf{S}}} \simeq \tilde{\tilde{\mathbf{S}}} \mathbf{P}_{S^{*}} \simeq \tilde{\mathbf{S}} \mathbf{P}_{S^{*}} \mathbf{P}_{S^{*}}=\tilde{\mathbf{S}} \mathbf{P}_{S^{*}} \simeq \tilde{\tilde{\mathbf{S}}}
$$




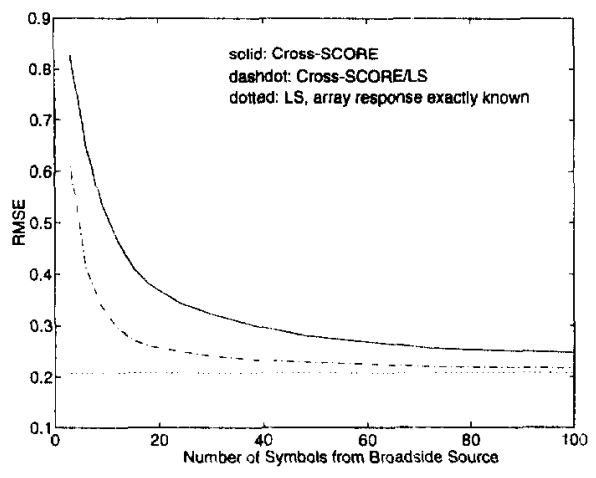

Figure 1: Performance of Blind/LS vs. Cross-SCORE

\subsection{A Simulation Example}

In this example, a 6 -sensor $\lambda / 2$ uniform linear array (ULA) with two $10 \mathrm{~dB}$ SNR signals incident from $\left[0^{\circ}, 12^{\circ}\right]$ was simulated. Both signals were BPSK with full raised-cosine pulse shapes and baud rates of 4 and 6 samples per symbol, respectively, and hence both signals were uncorrelated. An initial blind estimate of the signals was generated using Cross-SCORE [1] and the baud rate features of the signals. The root-MSE of Cross-SCORE based on 100 independent trials is plotted in Figure 1 (solid line) as a function of the number of symbols (measured with respect to the source at $\left.0^{\circ}\right)$ used to calculate the weight vectors. The dash-dot curve shows the root-MSE that results from refining the initial Cross-SCORE estimate using the LS steps described by (4) and (5). The dotted line shows the theoretical root-MSE performance of the LS algorithm assuming a perfectly known array manifold (e.g., see [4]). Note that we are able to achieve performance comparable to the known-array-response case even though this information was not used by the algorithm. As expected, the performance gain between Cross-SCORE alone and the LS refinements is greatest for small data sets, and the improvement decreases as Cross-SCORE is able to converge towards its limiting solution.

\section{A Decision Directed Approach}

We assume in this section that the signals received by the array are digitally modulated, with known modulation properties (i.e., pulse shape, symbol rate, etc.). The algorithm presented in this section exploits the fact that given the sequence of (synchronized) transmitted symbols, it is possible to perfectly reconstruct a noisefree replica of the original signal. If only an estimate of the symbol sequence is available (e.g., based on prelim- inary bit decisions), the reconstructed signal will differ from the original signal only where incorrect bit decisions have been made. Furthermore, the reconstructed signal could be used as a reference for computing $\mathbf{R}_{x}$ s and hence an approximation to $\mathbf{W}_{M S E}$. An algorithm based on this idea is outlined below:

1. Obtain an initial estimate of the signal by either a conventional DOA-based or blind copy approach. Demodulate the signal to estimate the transmitted bits, and use the bit decisions to generate an improved estimate of the transmitted signal. Denote this estimate as $\hat{\mathbf{S}}_{0}$.

2. Compute the MMSE signal copy vectors

$$
\hat{\mathbf{W}}=\hat{\mathbf{R}}_{x x}^{-1} \hat{\mathbf{R}}_{x \tilde{s}_{0}},
$$

where $\hat{\mathbf{R}}_{x x}$ and $\hat{\mathbf{R}}_{x \hat{s}}$ are sample averages.

3. Compute the signal estimate $\hat{\mathbf{S}}=\hat{\mathbf{W}}^{*} \mathbf{X}$

4. Demodulate $\hat{\mathbf{S}}$ to obtain an estimate of the transmitted symbols. For the $n^{\text {th }}$ iteration, let the estimate of the transmitted symbols be denoted by $\mathbf{I}_{n}$

5. Using $\mathbf{I}_{n}$ as the modulating symbol stream, reconstruct an estimate of the transmitted signal. Denote this estimate as $\hat{\mathbf{S}}_{n}$

6. Set $\hat{\mathbf{S}}_{0}=\hat{\mathbf{S}}_{n}$.

7. Repeat steps 2 to 6 until $\mathbf{I}_{n}=\mathbf{I}_{n+1}$.

For simplicity, we are neglecting channel effects on the shape of the transmitted pulses, and we are assuming that both carrier and symbol synchronization may be achieved. The performance degradation due to synchronization mismatch can be calculated exactly as in the single channel case, and thus will not be explicitly addressed in our analysis below. Achieving synchronization for multiple co-channel signals is not a trivial task, and probably requires that some degree of spatial discrimination be used in obtaining the initial signal estimates. As $\hat{\mathbf{W}}$ is updated at each iteration, the ability to spatially separate the signals will improve, and consequently so will the ability to attain synchronization. Thus, in practice, steps 3 and 4 of the algorithm would probably include an additional operation where carrier and symbol timing are reacquired.

\subsection{Performance Analysis for BPSK Signals}

For BPSK signals, the $i^{\text {th }}$ element of $s(t)$ may be expressed as $s_{i}(t)= \pm s_{i} p(t-n T) e^{j \phi_{i}}$ for some integer $n$, where $p(t)$ is the (real-valued) pulse shape, $T$ is the baud interval, $s_{i}$ is the (real-valued) amplitude of the signal, and $\phi_{i}$ is some phase shift that we presume to be known 
through proper synchronization. Assume that we have applied some algorithm to obtain an initial estimate $\hat{\mathbf{S}}$, and assume for simplicity that the BER of each of the initial signal estimates (the rows of $\hat{\mathbf{S}}$ ) is given by $b$. Under these assumptions, it is shown in [5] that if the noise is temporally white and Gaussian and the SNR is sufficiently high, then $\hat{\mathbf{s}}(t)$ is normally distributed with mean and variance

$$
\begin{aligned}
& \mathbf{m} \simeq(1-2 b) \mathbf{s}(t) \\
& \mathbf{\Sigma} \simeq \sigma_{n}^{2}(1-2 b)^{2}\left(\mathbf{A}^{*} \mathbf{A}\right)^{-1} .
\end{aligned}
$$

Assuming a binary pulse shape of unit power, the output of an optimal matched filter receiver used to demodulate the $i^{\text {th }}$ signal $\hat{\hat{s}}_{i}(t)$ at symbol $n$ will be a complex Gaussian random variable with mean $\bar{m}_{s_{i}}=$ $\pm(1-2 b) s_{i}$ and variance $\sigma_{s_{i}}^{2}=\Sigma_{i i}$. Since the bit decision is based only on the sign of the matched filter output, the BER can be computed as

$$
\mathrm{BER}_{i}=\frac{1}{2} \Phi\left(\frac{\vec{m}_{s_{i}}}{\sigma_{s_{i}}}\right)
$$

where $\Phi()$ is the complementary error function. It is easily shown that

$$
\left(\mathbf{A}^{*} \mathbf{A}\right)_{i i}^{-1}=\frac{1}{\mathbf{a}^{*}\left(\theta_{i}\right) \mathbf{P}_{B_{i}}^{1} \mathbf{a}\left(\theta_{i}\right)},
$$

where $\mathbf{B}_{\boldsymbol{i}}$ is the matrix consisting of all the columns of A except $\mathbf{a}\left(\theta_{i}\right)$. Consequently,

$$
\sigma_{s_{i}}^{2}=\mathbf{\Sigma}_{i i} \simeq \sigma_{n}^{2}(1-2 b)^{2} \frac{1}{\mathbf{a}_{i}^{*} \mathbf{P}_{B_{i}}^{1} \mathbf{a}_{i}}
$$

where $\mathbf{a}_{i}=\mathbf{a}\left(\theta_{i}\right)$, and hence

$$
\frac{\vec{m}_{s_{i}}}{\sigma_{s_{i}}}=\frac{(1-2 b) s_{i} \sqrt{\mathbf{a}_{i}^{*} \mathbf{P}_{B_{i}}^{\perp} \mathbf{a}_{i}}}{(1-2 b) \sigma_{n}}=\sqrt{S N R_{i} \times \mathbf{a}_{i}^{*} \mathbf{P}_{B_{i}}^{\perp} \mathbf{a}_{i}},
$$

so our analysis predicts that the above decision directed algorithm will result in a terminal BER given by

$$
\mathrm{BER}_{i}=\frac{1}{2} \Phi\left(\sqrt{S N R_{i} \times \mathbf{a}_{i}^{*} \mathbf{P}_{B_{i}}^{\perp} \mathbf{a}_{i}}\right)
$$

for signal $i$. The key quantities in determining the terminal BER are thus the SNR and the degree of spatial coherence between the signals. Note that for this case, the terminal BER is independent of the BER of the initial signal estimate. Simulations have shown this result to hold even for initial BERs as high as $40 \%$.

\subsection{A Simulation Example}

In this example, a scenario involving two baseband $0 \mathrm{~dB}$ SNR BPSK signals received by a five element $\lambda / 2$

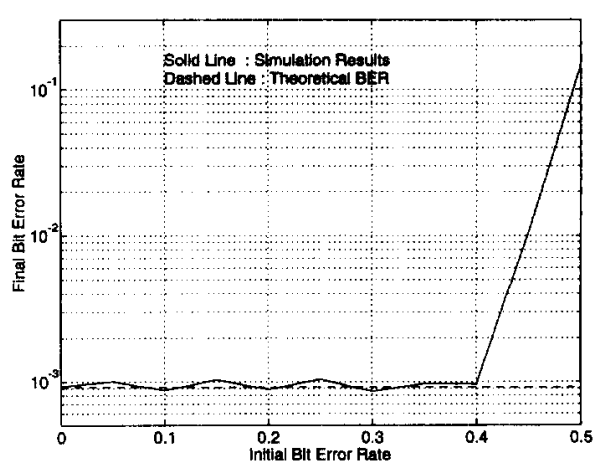

Figure 2: BER performance of decision directed algorithm vs. initial BER

ULA was simulated. The DOAs of the signals were $0^{\circ}, 20^{\circ}$, and the signals were assumed to be uncorrelated, have the same baud rate ( 6 samples per symbol), and be symbol synchronized with one another (worst case situation for signal separation). In this case the initial estimate of each signal was obtained by taking the actual signal and artificially generating bit errors at various BER. A total of 500 trials ( 500 symbols per trial) were conducted for each initial BER, and the final BER of the decision directed algorithm was computed. The results are plotted in Figure 2 along with the terminal BER predicted by (14). Our theoretical BER calculation accurately matches the actual terminal BER, and correctly predicts that the final error rate is independent of the initial error rate for initial BERs as high as 0.4 .

\section{References}

[1] B. Agee, S. Schell, and W. Gardner, "Spectral Self-Coherence Restoral: A New Approach to Blind Adaptive Signal Extraction Using Antenna Arrays", Proceedings of the IEEE, 78(4):753-767, April 1990.

[2] R. Gooch and J. Lundel, "The CM Array: An Adaptive Beamformer for Constant Modulus Signals", In Proc. IEEE ICASSP, pages 2523-2526, 1986.

[3] S. Talwar, M. Viberg, and A. Paulraj, "Blind Estimation of Multiple Co-Channel Digital Signals Arriving at an Antenna Array", In Proc. $27^{\text {th }}$ A silomar Conference on Signals, Systems, and Computers, Asilomar, CA, November 1993.

[4] J. Yang and A. Swindlehurst, "Signal Copy with Array Calibration Errors", In Proc. $27^{\text {th }}$ Asilomar Conference on Signals, Systems, and Computers, Asilomar, CA., November 1993.

[5] A. Swindlehurst, S. Daas, and J. Yang, "A Decision Directed Approach for Signal Copy using Antenna Arrays", IEEE Tranc. on Sig. Proc., October 1993, (in review) 